\title{
A GENERAL ENERGY FORMULA
}

\author{
KATHRYN E. HARE, PARASAR MOHANTY and MARIA ROGINSKAYA*
}

\begin{abstract}
An analogue of the formula relating the Riesz energy of a measure on the torus with a weighted $l^{2}$ norm of its Fourier transform is given for energies arising from more general kernels. This involves estimating the Fourier transform of the kernel. The formula is used to study certain measures of (classical) energy dimension zero.
\end{abstract}

\section{Introduction}

There is an important and useful relationship between the $t$-Riesz energy of a measure $\mu$ defined on the circle, $I_{t}(\mu)$, and its Fourier transform

$$
I_{t}(\mu) \equiv \iint \frac{d \mu(x) d \mu(y)}{|x-y|^{t}} \sim|\widehat{\mu}(0)|+\sum_{n \in Z \backslash\{0\}}|n|^{t-1}|\widehat{\mu}(n)|^{2} .
$$

One reason for the interest in the energy of a measure is that if $I_{t}(\mu)<\infty$, then the Hausdorff dimension of $\mu$ is at least $t$. Both this formula and the analogous classical formula relating the energy of a measure on $\mathrm{R}^{n}$ with its Fourier transform have been used to study a variety of things, including distance sets, the average rate of decay of the Fourier transform and the singularity of Riesz products (c.f. [8], [13, ch. 12], [16] and the references cited therein).

The $t$-energy has been generalized in different ways. Kahane in [12] considered replacing the kernel $|x|^{-t}$ by $|\log | x||$ and established a similar result to (1.1) by proving that the $n$ 'th Fourier coefficient of $|\log | x||$ was comparable to $1 /|n|$. In [9] the kernel $|x|^{-t}$ was replaced by the function $|x|^{-1}|\log | x||^{s}$. This gave the possibility of distinguishing measures of energy dimension one, the maximum possible.

Here we replace $|x|^{-t}$ by more general kernels, including $\left.|x|^{-\beta}|\log | x\right|^{\alpha}$ for $0<\beta<1$, or $\beta=0$ and $\alpha>0$, and establish an energy formula analogous to (1.1). The weights on the right hand side of the formula will be the Fourier transform of the kernel, so to have a useful formula one needs good

\footnotetext{
* This work was supported in part by NSERC. The second and third authors thank the University of Waterloo for its hospitality.

Received June 11, 2004; in revised form April 25, 2006.
} 
estimates for the transform. The main contribution of the paper, which may be of independent interest, is to derive an elementary procedure for approximating the Fourier transform of a class of kernels that satisfy certain technical (but not overly restrictive) conditions.

Our formula provides a tool to study measures of dimension zero, but for which

$$
\iint|\log | x-y||^{\alpha} d \mu(x) d \mu(y)<\infty
$$

for some $\alpha>0$. Cantor measures supported on Cantor sets with ratios tending to zero at a suitable rate or smooth measures on 'flat' curves (c.f. [1], [7]) are examples of measures with this property. In particular, we investigate the connection with Lipschitz-like properties of the measure and the mapping properties of Lorentz or Orlicz-improving measures.

\section{Refined Energy}

\subsection{Notation and Terminology}

Throughout the paper the circle $T$ will be the interval $[-1 / 2,1 / 2]$. When we speak of a measure we mean a finite, regular, positive Borel measure on $\mathrm{T}$. We assume Lebesgue measure on T is normalized so that the Fourier transform of an even, integrable function is given by

$$
\widehat{\phi}(n)=\int_{0}^{1 / 2} \phi(x) \cos 2 \pi n x d x .
$$

Recall that a positive function $\psi$ is said to satisfy the doubling condition if there are positive constants $c, C$ such that

$$
c \psi(2 x) \leq \psi(x) \leq C \psi(2 x) \text { for all } x .
$$

By a kernel, we will mean a function $\phi$ defined on the circle that is positive, even, integrable, convex, decreasing for $x>0$, and satisfies the doubling condition.

Given a kernel $\phi$, the $\phi$-energy of a measure $\mu$ is given by

$$
I_{\phi}(\mu)=\iint \phi(x-y) d \mu(x) d \mu(y)
$$

where $x-y$ is understood to be the group operation. 


\subsection{A Fourier transform formula for $\phi$-energy}

We first observe that kernels have positive Fourier transform.

LEMMA 2.1. If $f$ is even, convex, decreasing and positive, then $\hat{f}(n) \geq 0$ for all $n$.

Proof. Notice that

$$
\begin{array}{rl}
\int_{0}^{1 / 2} & f(x) \cos 4 \pi x d x \\
= & \int_{0}^{1 / 8}\left[f(x)-f\left(\frac{1}{4}-x\right)-f\left(x+\frac{1}{4}\right)+f\left(\frac{1}{2}-x\right)\right] \cos 4 \pi x d x .
\end{array}
$$

The convexity of $f$ implies that $f(-x+1 / 2)-f(x+1 / 4) \geq f(-x+1 / 4)-$ $f(x)$, hence

$$
\int_{0}^{1 / 2} f(x) \cos 4 \pi x d x \geq 0 .
$$

Now, subdivide the interval [0,1/2] into the periods of $\cos 2 n \pi x$. The previous inequality implies that on each of these subintervals $\int f(x) \cos 2 \pi n x$ is positive. If $n$ is odd there will be a half-period remaining, but cosine is positive on $[0, \pi / 2]$ and $f$ is decreasing, so this portion of the integral is positive as well. Hence $\hat{f}(n) \geq 0$.

Proposition 2.2. If $\phi$ is a kernel and $\mu$ a measure on $\mathrm{T}$, then

$$
I_{\phi}(\mu) \leq \sum_{n=-\infty}^{\infty} \widehat{\phi}(n)|\widehat{\mu}(n)|^{2}
$$

Proof. The proof is similar to that given in [8] for the $t$-energy. Let $\psi$ be a non-negative, continuous, even function supported on $[-1 / 6,1 / 6]$. Assume $\psi$ satisfies $\int \psi=1$ and $\widehat{\psi} \geq 0$. Let $\left\{\psi_{\varepsilon}\right\}_{\varepsilon>0}$ be the approximate identity where $\psi_{\varepsilon}(x)=\varepsilon^{-1} \psi(x / \varepsilon)$. Note that $\psi_{\varepsilon}$ is supported on $[-\varepsilon / 6, \varepsilon / 6]$.

By Fatou's lemma

$$
\iint \phi(x-y) d \mu(x) d \mu(y) \leq \liminf _{\varepsilon \rightarrow 0} \iint \psi_{\varepsilon} * \phi(x-y) d \mu(x) d \mu(y) .
$$

Since $\psi_{\varepsilon} * \phi$ is a continuous function on the torus, Parseval's theorem (applied first to the exterior integral) gives

$$
\iint\left(\psi_{\varepsilon} * \phi\right)(x-y) d \mu(x) d \mu(y)=\sum_{n} \widehat{\psi_{\varepsilon}}(n) \widehat{\phi}(n)|\widehat{\mu}(n)|^{2},
$$


and the sum converges to $\sum \widehat{\phi}(n)|\widehat{\mu}(n)|^{2}$ as $\varepsilon \rightarrow 0$ since $\widehat{\psi_{\varepsilon}}(n) \rightarrow 1$ pointwise from below.

THEOREM 2.3. Suppose $\phi$ is a kernel which satisfies the condition

$$
\frac{1}{z} \int_{0}^{z} \phi(x) d x \leq B \phi(z)
$$

for positive $z$ in a neighbourhood of 0 . Then for any measure $\mu$ on $\mathrm{T}$,

$$
I_{\phi}(\mu)=\sum_{n=-\infty}^{\infty} \widehat{\phi}(n)|\widehat{\mu}(n)|^{2} .
$$

Proof. In view of the previous proposition we only need to prove that

$$
I_{\phi}(\mu) \geq \sum_{n=-\infty}^{\infty} \widehat{\phi}(n)|\widehat{\mu}(n)|^{2}
$$

and this is trivially true if $I_{\phi}(\mu)$ is infinite. Thus we can assume the function $(x, y) \mapsto \phi(x-y)$ belongs to $L^{1}(\mu \times \mu)$.

We will continue to use the notation of the previous proof. It will be enough to prove that

$$
\iint \phi(x-y) d \mu(x) d \mu(y)=\lim _{\varepsilon \rightarrow 0} \iint \psi_{\varepsilon} * \phi(x-y) d \mu(x) d \mu(y) .
$$

To justify this we will check that there is a constant $\kappa$ such that $\psi_{\varepsilon} * \phi(z) \leq$ $\kappa \phi(z)$ for $z \neq 0$. Since $\psi_{\varepsilon} * \phi \rightarrow \phi$ pointwise, Lebesgue's theorem will then imply (2.3).

As the functions are even we can assume $z>0$ and we write $\psi_{\varepsilon} * \phi(z)$ as

$$
\int_{z / 2}^{2 z} \psi_{\varepsilon}(x) \phi(z-x) d x+\int_{x \notin(z / 2,2 z)} \psi_{\varepsilon}(x) \phi(z-x) d x .
$$

Since $\phi(z)$ is even and decreasing for $z>0, \phi(z-x) \leq \phi(z / 2)$ when $x \notin(z / 2,2 z)$. Hence the doubling condition ensures that the second integral is bounded by $\kappa_{1} \phi(z)$ (for a suitable constant $\kappa_{1}$ ).

The first integral is zero if $\varepsilon<z / 2$. As $\psi$ is bounded, if $\varepsilon \geq z / 2$

$$
\int_{z / 2}^{2 z} \psi_{\varepsilon}(x) \phi(z-x) d x \leq \frac{\kappa_{2}}{z} \int_{0}^{z} \phi(t) d t \leq \kappa_{2} B \phi(z) .
$$

Together these estimates establish that $\psi_{\varepsilon} * \phi(z) \leq \kappa \phi(z)$. 
COROLlaRY 2.4. Suppose that $\phi$ is a kernel, $\lim _{x \rightarrow 0} x \phi(x)=0$ and there is some $\varepsilon>0$ such that $-x \phi^{\prime}(x) \leq(1-\varepsilon) \phi(x)$ for all $x \neq 0$. Then (2.2) holds.

Proof. An integration by parts argument allows one to verify (2.1).

\subsection{Estimating the Fourier transform}

Of course, the previous results are mainly of interest if one can estimate $\widehat{\phi}$. In this subsection we show that for a reasonable class of kernels there is a simple way to estimate the Fourier transform.

Notation. When we write $f \sim g$ we mean there are positive constants $a, b$ such at $a f \leq g \leq b f$.

THEOREM 2.5. Suppose $\phi$ is a kernel that is smooth away from the origin, $-\phi^{\prime}$ satisfies the doubling condition and $\lim _{x \rightarrow 0} x^{2} \phi^{\prime}(x)=0$. Suppose also that either

(i) for some $\varepsilon>0,0 \leq\left(x \phi^{\prime}(x)\right)^{\prime} \leq(1-\varepsilon)\left(-\phi^{\prime}(x)\right)$ for all small $x>0$, or

(ii) $\left(x \phi^{\prime}(x)\right)^{\prime} \leq 0$ and $-\phi^{\prime}(x) \geq-3 \phi^{\prime}(4 x)$ for small $x>0$.

Then there is a positive integer $n_{0}$ such that

$$
\widehat{\phi}(n) \sim-\frac{\phi^{\prime}(1 /|n|)}{n^{2}} \quad \text { for } \quad|n| \geq n_{0} .
$$

REMARK 2.1. There are other possible assumptions one can make than $-\phi^{\prime}(x) \geq-3 \phi^{\prime}(4 x)$, but this is a convenient choice. Before proving the theorem we give several corollaries and examples.

Corollary 2.6. Suppose $\phi$ satisfies the conditions of both Theorems 2.3 and 2.5. If $\phi \neq 0$ a.e. then

$$
I_{\phi}(\mu) \sim \sum_{n \neq 0}-\frac{1}{n^{2}} \phi^{\prime}\left(\frac{1}{|n|}\right)|\widehat{\mu}(n)|^{2}+|\widehat{\mu}(0)|^{2} .
$$

REMARK 2.2. We are often only interested in whether $I_{\phi}(\mu)$ is finite or infinite, so having equivalence here, rather than equality, is not significant.

Proof of Corollary 2.6. As $\phi$ is decreasing away from zero there must be a neighbourhood on which it is bounded away from zero. Thus $I_{\phi}(\mu) \geq a\|\mu\|^{2}$ 
for some constant $a>0$. Since it is also the case that $\left.\sum_{|n|<n_{0}} \widehat{\phi}(n)\right)|\widehat{\mu}(n)|^{2} \leq$ $b\|\mu\|^{2}=b|\widehat{\mu}(0)|^{2}$ for large enough $b$ (and $n_{0}$ as in the theorem), we have

$$
I_{\phi}(\mu) \sim \sum_{|n| \geq n_{0}} \widehat{\phi}(n)|\widehat{\mu}(n)|^{2}+|\widehat{\mu}(0)|^{2} .
$$

But for suitably large $b$,

$$
0 \leq \frac{-\phi^{\prime}(1 /|n|)}{n^{2}}|\widehat{\mu}(n)|^{2} \leq b|\widehat{\mu}(0)|^{2} \quad \text { for all } n \neq 0,
$$

hence

$$
I_{\phi}(\mu) \sim \sum_{n \neq 0}-\frac{1}{n^{2}} \phi^{\prime}\left(\frac{1}{|n|}\right)|\widehat{\mu}(n)|^{2}+|\widehat{\mu}(0)|^{2} .
$$

EXAMPLE 2.1. If $-x \phi^{\prime}=\psi(\phi(x))$ then the assumption of case (i) is equivalent to $0 \leq \psi^{\prime}(x) \leq 1-\varepsilon$ for large $x$. The classical formula relating energy and the Fourier transform, (1.1), can be recovered by putting $\phi(x)=|x|^{-t}$ for $0<t<1$ and $\psi(x)=t x$.

Notation. Denote $\log ^{+}|n|=\max \{1, \log |n|\}$.

Corollary 2.7. Suppose $\phi(x)=|x|^{-\beta}|\log | x||^{\alpha}$ for $0<\beta<1$. Then

$$
\iint \frac{|\log | x-y||^{\alpha}}{|x-y|^{\beta}} d \mu(x) d \mu(y) \sim \sum_{n \neq 0} \frac{\left(\log ^{+}|n|\right)^{\alpha}}{|n|^{1-\beta}}|\widehat{\mu}(n)|^{2}+|\widehat{\mu}(0)|^{2} .
$$

ProOF. Just check that the conditions of case (i) of the theorem are satisfied.

Corollary 2.8. If $\alpha>0$, then

$$
\iint|\log | x-y||^{\alpha} d \mu(x) d \mu(y) \sim \sum_{n \neq 0} \frac{\left(\log ^{+}|n|\right)^{\alpha-1}}{|n|}|\widehat{\mu}(n)|^{2}+|\widehat{\mu}(0)|^{2} .
$$

PROOF. If $\alpha \geq 1$ the assumption of case (i) can be verified by taking $\psi(t)=$ $\alpha t^{(\alpha-1) / \alpha}$ (following the notation of the example above).

If $\alpha<1$ then the requirements of case (ii) are satisfied.

Proof of Theorem 2.5. First, we will prove that there is some constant $a>0$ and integer $n_{0}$ such that

$$
\widehat{\phi}(n) \geq a\left(-\frac{\phi^{\prime}(1 /|n|)}{n^{2}}\right) \quad \text { for } \quad|n| \geq n_{0} .
$$


As $\phi$ is even it suffices to consider $n>0$.

We begin by defining a new, even function

$$
\phi_{1}(x)= \begin{cases}\phi(x)+(-x+1 / 4 n) \phi^{\prime}(1 / 4 n)-\phi(1 / 4 n) & \text { for } x \in[0,1 / 4 n] \\ 0 & \text { for } x \in[1 / 4 n, 1 / 2] .\end{cases}
$$

The difference, $\phi-\phi_{1}$, is even, convex, positive and integrable, thus $\widehat{\phi}(n) \geq$ $\widehat{\phi}_{1}(n)$. From the Fourier transform formula we have

$$
\widehat{\phi}_{1}(n)=\int_{0}^{1 / 2} \phi_{1}(x) \cos 2 \pi n x d x \geq \frac{\sqrt{2}}{2} \int_{0}^{1 / 8 n} \phi_{1}(x) d x .
$$

Define a second even function by

$$
\phi_{2}(x)= \begin{cases}\phi(x)+(-x+1 / 8 n) \phi^{\prime}(1 / 8 n)-\phi(1 / 8 n) & \text { for }[0,1 / 8 n] \\ 0 & \text { for }[1 / 8 n, 1 / 2] .\end{cases}
$$

The convexity of $\phi$ allows one to verify that $\phi_{1} \geq \phi_{2}$, thus it will be enough to show that

$$
\int_{0}^{1 / 8 n} \phi_{2} \geq a\left(-\frac{\phi^{\prime}(1 / n)}{n^{2}}\right)
$$

for $n$ suitably large.

After integrating by parts (twice) we obtain,

$$
\begin{aligned}
\int_{0}^{1 / 8 n} \phi_{2} & =-\int_{0}^{1 / 8 n} x \phi^{\prime}(x) d x+\frac{\phi^{\prime}(1 / 8 n)}{2(8 n)^{2}} \\
& =-\frac{\phi^{\prime}(1 / 8 n)}{(8 n)^{2}}+\int_{0}^{1 / 8 n} x\left(x \phi^{\prime}(x)\right)^{\prime} d x+\frac{\phi^{\prime}(1 / 8 n)}{2(8 n)^{2}} .
\end{aligned}
$$

Suppose (i) holds. Since $\left(x \phi^{\prime}\right)^{\prime} \geq 0$ for small $x$ and the doubling condition implies $-\phi^{\prime}(1 / 8 n) \sim-\phi^{\prime}(1 / n)$, the desired bound easily follows.

If assumption (ii) holds we note that

$$
\begin{aligned}
-\int_{0}^{1 / 8 n} x \phi^{\prime}(x) d x+\frac{\phi^{\prime}(1 / 8 n)}{2(8 n)^{2}} & \geq-\int_{1 / 32 n}^{1 / 8 n} x \phi^{\prime}(x) d x+\frac{\phi^{\prime}(1 / 8 n)}{2(8 n)^{2}} \\
& \geq-\frac{\phi^{\prime}(1 / 32 n)}{32 n}\left(\frac{1}{8 n}-\frac{1}{32 n}\right)+\frac{\phi^{\prime}(1 / 8 n)}{2(8 n)^{2}} \\
& \geq-\frac{\phi^{\prime}(1 / 8 n)}{(32 n)^{2}} \quad \text { for } n \text { sufficiently large }
\end{aligned}
$$

and the doubling condition completes the argument. 
Now we will show the upper bound on $\widehat{\phi}(n)$. Similar arguments to those used in Lemma 2.1 show that

$$
\int_{1 / 2 n}^{1 / 2} \phi(x) \cos 2 n \pi x d x \leq 0 .
$$

Thus

$$
\begin{aligned}
\widehat{\phi}(n) & \leq \int_{0}^{1 / 2 n} \phi(x) \cos 2 n \pi x d x \\
& =\int_{0}^{1 / 4 n}(\phi(x)-\phi(-x+1 / 2 n)) \cos 2 n \pi x d x \\
& \leq \int_{0}^{1 / 4 n}(\phi(x)-\phi(-x+1 / 2 n)) d x .
\end{aligned}
$$

Since $\phi^{\prime}$ is increasing,

$$
\int_{0}^{1 / 4 n} x \phi^{\prime}(-x+1 / 2 n) d x \geq \phi^{\prime}(1 / 4 n) \int_{0}^{1 / 4 n} x d x=\frac{\phi^{\prime}(1 / 4 n)}{2(4 n)^{2}} .
$$

Integrating by parts and simplifying gives

$$
\begin{aligned}
\int_{0}^{1 / 4 n} & (\phi(x)-\phi(-x+1 / 2 n)) d x \\
= & \left.x \phi(x)\right|_{0} ^{1 / 4 n}-\int_{0}^{1 / 4 n} x \phi^{\prime}(x) d x \\
& \quad-\left[\left.x \phi(-x+1 / 2 n)\right|_{0} ^{1 / 4 n}+\int_{0}^{1 / 4 n} x \phi^{\prime}(-x+1 / 2 n)\right] \\
\leq & \int_{0}^{1 / 4 n}-x \phi^{\prime}(x) d x-\frac{\phi^{\prime}(1 / 4 n)}{2(4 n)^{2}} .
\end{aligned}
$$

In case (ii) the function $-x \phi^{\prime}(x)$ is increasing and thus

$$
\begin{aligned}
\int_{0}^{1 / 4 n}-x \phi^{\prime}(x) d x-\frac{\phi^{\prime}(1 / 4 n)}{2(4 n)^{2}} & \leq-\frac{\phi^{\prime}(1 / 4 n)}{(4 n)^{2}}-\frac{\phi^{\prime}(1 / 4 n)}{2(4 n)^{2}} \\
& \leq-\frac{c \phi^{\prime}(1 / n)}{n^{2}} .
\end{aligned}
$$

In case (i) we apply integration by parts a second time to obtain

$$
-\int_{0}^{1 / 4 n} x \phi^{\prime}(x) d x=-\frac{\phi^{\prime}(1 / 4 n)}{(4 n)^{2}}+\int_{0}^{1 / 4 n} x\left(x \phi^{\prime}(x)\right)^{\prime} d x .
$$


Substituting in the bound $\left(x \phi^{\prime}(x)\right)^{\prime} \leq(1-\varepsilon)\left(-\phi^{\prime}(x)\right)$ and simplifying gives

$$
-\int_{0}^{1 / 4 n} x \phi^{\prime}(x) d x \leq-\frac{c \phi^{\prime}(1 / 4 n)}{(4 n)^{2}} .
$$

If we use this estimate in (2.4) and apply the doubling condition to $-\phi^{\prime}$ the desired result is obtained.

Given an even sequence of positive weights, $\left\{w_{n}\right\}$, it can be of interest to know if the formula $\sum w_{n}^{2}|\widehat{\mu}(n)|^{2}$ corresponds to some general energy. We will give a constructive method for finding a kernel assuming that the weights form the sequence of Fourier coefficients of a function $f$ which is even, positive, integrable, convex, decreasing and smooth away from the origin.

Proposition 2.9. Suppose $f$ is a kernel that is smooth away from the origin. Let $K_{f}$ be the even function defined by

$$
K_{f}(x)=\int_{x}^{1}\left(y f^{\prime}(y)\right)^{2} d y \quad \text { for } \quad x \geq 0 .
$$

Then

$$
\iint K_{f}(x-y) d \mu(x) d \mu(y) \sim \sum_{n \neq 0}|\hat{f}(n) \widehat{\mu}(n)|^{2}+|\widehat{\mu}(0)|^{2}
$$

provided $-f^{\prime}$ satisfies the doubling condition, $\lim _{x \rightarrow 0} x^{2} f^{\prime}(x)=0,-\frac{3}{2} f^{\prime} \leq$ $x f^{\prime \prime} \leq(2-\varepsilon)\left(-f^{\prime}\right)$ for some $\varepsilon>0$ and $\int_{0}^{z} K_{f}(x) d x \leq B z K_{f}(z)$.

Moreover,

$$
\hat{f}(n) \sim-\frac{1}{n^{2}} f^{\prime}(1 / n) \quad \text { and } \quad \widehat{K_{f}}(n) \sim\left(\frac{1}{n^{2}} f^{\prime}(1 / n)\right)^{2} .
$$

Proof. We check that $f$ and $K_{f}$ satisfy the conditions of Theorem 2.5. Notice that $K_{f}^{\prime}=-\left(x f^{\prime}(x)\right)^{2} \leq 0$, so clearly $K_{f}$ is decreasing. Integrability can be checked using the integrability of $f$; convexity from the observation that $x f^{\prime \prime} \geq-f^{\prime}$. The doubling properties and limiting behaviour of $x K_{f}(x)$ and $x^{2} K_{f}^{\prime}$ are easily checked. It is also a routine matter to verify that condition (i) of Theorem 2.5 is satisfied for both functions $f$ and $K_{f}$.

REMARK 2.3. One can obtain a similar proposition based on condition (ii) of Theorem 2.5. It would be interesting to find a general result. 


\section{Applications}

\subsection{Fine Hausdorff dimension}

The Hausdorff and energy dimensions of a measure (for definitions see [6] and [8]) are two ways to quantify singularity. As there are continuous measures of dimension zero it is of interest to refine these notions of dimension.

Suppose we are given a kernel $\phi$ with $\lim _{x \rightarrow 0} \phi(x)=\infty$. If we put $h(x)=$ $1 / \phi(x)$, then we may define the $h$-Hausdorff measure of a Borel set $E$ as in [14] by $h(E)=\lim _{\delta \rightarrow 0} h_{\delta}(E)$ where

$$
h_{\delta}(E)=\inf \left\{\sum h\left(\operatorname{diam} U_{j}\right): \bigcup U_{j} \supseteq E, \operatorname{diam} U_{j} \leq \delta\right\} .
$$

The case $\phi(x)=|x|^{-t}$ gives the classical $t$-Hausdorff measure.

Definition 3.1. Put $h^{s}(x)=|\log | x||^{-s}$. For a measure $\mu$ of Hausdorff dimension zero we define the Fine 0-Hausdorff dimension of $\mu$ by

$$
\text { Fine }_{0} \operatorname{dim}_{H} \mu=\inf \left\{\inf \left\{s>0: h^{s}(F)<\infty\right\}: \mu(F)>0\right\} .
$$

For a measure $\mu$ of energy dimension zero define the Fine 0-energy dimension of $\mu$ by

$$
\text { Fine }_{0} \operatorname{dim}_{e} \mu=\sup \left\{s>0: I_{1 / h^{s}}(\mu)<\infty\right\} .
$$

The same arguments as given in the classical case (see [5, ch. 4]) show that if there exists a measure $\mu$ on $E$ such that $I_{1 / h}(\mu)<\infty$, then $h(E)=\infty$. Thus Fine $\operatorname{dim}_{H} \mu \geq$ Fine $_{0} \operatorname{dim}_{e} \mu$.

\subsection{Lorentz and Lorentz-Zygmund improving measures}

It follows easily from Corollary 2.8 that if $|\widehat{\mu}(n)| \leq O(\log |n|)^{-\varepsilon}$ for some $\varepsilon>0$, then Fine $\operatorname{dim}_{e} \mu \geq 2 \varepsilon$. In this section we will study other classes of measures which have positive Fine 0-Hausdorff dimension.

A natural generalization of the classical Banach spaces $L^{p}$ are the LorentzZygmund spaces, $L^{p, q}(\log L)^{\alpha}$ for $0<p, q<\infty,-\infty<\alpha<\infty$, where the quasi-norm is given by

$$
\|f\|_{p, q, \alpha}=\left(\int_{0}^{1}\left[t^{1 / p}(1-\log t)^{\alpha} f^{*}(t)\right]^{q} \frac{d t}{t}\right)^{1 / q}
$$

for $f^{*}$ the decreasing rearrangement of $f$. The Banach space $L^{p}$ is the LorentzZygmund space with $p=q, \alpha=0$. The Lorentz space $L(p, q)$ is the LorentzZygmund space $L^{p, q}(\log L)^{0}$,

Lorentz-Zygmund spaces for $p, q=\infty$ are also defined, but these will not be of interest to us. 
There are various inclusions between the Lorentz-Zygmund spaces. We list some below:

$$
\begin{aligned}
& L^{p, q}(\log L)^{\alpha} \subset L^{p, q}(\log L)^{\beta} \text { if } \alpha>\beta ; \\
& L^{p_{1}, q_{1}}(\log L)^{\alpha_{1}} \subset L^{p_{2}, q_{2}}(\log L)^{\alpha_{2}} \text { if } p_{1}>p_{2} ; \\
& L^{p, q_{1}}(\log L)^{\alpha} \subset L^{p, q_{2}}(\log L)^{\beta} \text { if either } q_{1} \leq q_{2} \text { and } \alpha \geq \beta, \text { or } q_{1}>q_{2} \text { and } \\
& \alpha+1 / q_{1}>\beta+1 / q_{2} .
\end{aligned}
$$

In particular, this implies that

$$
L(2, q) \subset L^{2,2}(\log L)^{-\alpha} \quad \text { whenever } \quad \alpha>1 / 2-1 / q
$$

and, consequently,

$$
L(2, q) \subset L^{2} \text { if } q>2
$$

The Lorentz-Zygmund space, $L^{2,2}(\log L)^{\alpha}$, is also known to be equal to the Orlicz space $L^{\Phi}$ with $\Phi(x)=x^{2}(\log x)^{\alpha / 2}$. For proofs of these facts and other properties of Lorentz-Zygmund spaces we refer the reader to [3].

A measure is called $L^{p}$-improving if it acts by convolution as a bounded operator from $L^{p}$ to $L^{2}$ for some $p<2$. Singular examples include Riesz product measures and the Cantor measure. It was shown in [10] that $L^{p}$ improving measures have positive energy dimension.

But there are also examples of "improving" measures that have dimension zero.

DEFINITION 3.2. (i) A measure $\mu$ is said to be Lorentz-improving if under convolution $\mu$ maps a Lorentz space $L(2, q)$, for some $q>2$, into the (proper) subspace $L^{2}$.

(ii) A measure $\mu$ is said to be Lorentz-Zygmund improving if under convolution $\mu$ maps a Lorentz-Zygmund space $L^{2,2}(\log L)^{-\alpha}$, for some $\alpha>0$, into the (proper) subspace $L^{2}$.

Of course, Lorentz-Zygmund improving measures are always Lorentzimproving. Examples of Cantor measures which are Lorentz-improving, but have dimension zero, can be found in [7].

Proposition 3.1. Suppose $\mu$ is Lorentz-improving. Then $\mu$ has positive Fine 0-energy dimension.

Proof. Consider

$$
\sum_{n \neq 0} \frac{\left(\log ^{+}|n|\right)^{s-1}}{|n|}|\widehat{\mu}(n)|^{2}
$$


for $s>0$ and note that this sum is dominated by the square of the $L^{2}$ norm of $\mu * F$ where $\widehat{F}(n)=\left(\log ^{+}|n|\right)^{(s-1) / 2} / \sqrt{|n|}$. Such a function $F$ is known ([15]) to belong to the Lorentz space $L(2, q)$ for $q>2$ provided

$$
\sum_{n=1}^{\infty}\left(n^{-1 / 2}\left(\log ^{+} n\right)^{(s-1) / 2}\right)^{q} n^{q / 2-1}<\infty
$$

and this is the case if $s<1-2 / q$. Thus the Fine 0 -energy dimension is at least $1-2 / q$.

COROLlary 3.2. Suppose $|\widehat{\mu}(n)|$ decreases monotonically away from zero. Then $\mu$ is Lorentz-improving if and only if Fine $_{0} \operatorname{dim}_{e} \mu>0$.

Proof. Measures with monotonic Fourier transform are known ([7]) to be Lorentz-improving if and only if $|\widehat{\mu}(n)| \leq C\left(\log ^{+}|n|\right)^{s}$ for some $s<0$.

More can be said about the behaviour of Lorentz-Zygmund improving measures. It will be useful to first estimate the Lorentz-Zygmund norms of Dirichlet kernels and characteristic functions.

LEMMA 3.3. Let $\alpha>0$.

(i) There are positive constants $A, B$ such that for any interval I,

$$
A|I|(1-\log |I|)^{-2 \alpha} \leq\left\|\chi_{I}\right\|_{2,2,-\alpha}^{2} \leq B|I|(1-\log |I|)^{-2 \alpha} .
$$

(ii) There are positive constants $A, B$ such that if $D_{n}$ is the $n$ 'th Dirichlet kernel, then

$$
A n(\log n)^{-2 \alpha} \leq\left\|D_{n}\right\|_{2,2,-\alpha}^{2} \leq B n(\log n)^{-2 \alpha} .
$$

Proof. (i) We use the fact that $\chi_{I}^{*}=\chi_{[0,|I|]}$. Thus

$$
\left\|\chi_{I}\right\|_{2,2,-\alpha}^{2}=\int_{0}^{|I|}(1-\log t)^{-2 \alpha} d t .
$$

The claim follows from the fact that $(1-\log t)^{-2 \alpha}$ is an increasing function and $1-\log t \sim 1-\log t / 2$.

(ii) There are positive constants $A, B$ such that for $t \in[0,1 / 2]$,

$$
A n \chi_{[0,1 / 4(n+1 / 2)]} \leq D_{n}(t) \leq B\left(n \chi_{[0,1 /(n+1 / 2)]}+\frac{1}{t} \chi_{[1 /(n+1 / 2), 1 / 2]}\right)
$$

The estimates on the norms of characteristic functions shows that the $L^{2,2}(\log L)^{-\alpha}$ norms of both $n \chi_{[0,1 / 4(n+1 / 2)]}$ and $n \chi_{[0,1 /(n+1 / 2)]}$ are $O\left(n^{1 / 2}(\log n)^{-\alpha}\right)$. 
Observe that if $F=\frac{1}{t} \chi_{[1 /(n+1 / 2), 1 / 2]}$, then

$$
F^{*}(t)=B \frac{\chi_{[0,1 / 2-1 /(n+1 / 2)]}}{t+1 /(n+1 / 2)}
$$

Thus

$$
\|F\|_{2,2,-\alpha}^{2}=B \int_{0}^{1 / 2} \frac{(1-\log t)^{-2 \alpha}}{(t+1 /(n+1 / 2))^{2}} d t .
$$

For the integral from 0 to $1 / \sqrt{n}$, majorize $(1-\log t)^{-2 \alpha}$ by $c(\log n)^{-2 \alpha}$ and integrate to get a bound of $c n(\log n)^{-2 \alpha}$ (where the constant $c$ may change). For the integral from $1 / \sqrt{n}$ to $1 / 2$, just use the fact that $(1-\log t)^{-2 \alpha}$ is bounded and integrate the remaining term, $(t+1 /(n+1 / 2))^{-2}$. This gives a bound of $c \sqrt{n}$ which is even smaller. Therefore, $\|F\|_{2,2,-\alpha}^{2} \leq c n(\log n)^{-2 \alpha}$ and that completes the argument.

Proposition 3.4. If there is a constant $c$ and $\alpha>0$ such that for all $n$, $\left\|\mu * D_{n}\right\|_{2} \leq c\left\|D_{n}\right\|_{2,2,-\alpha}$, then Fine $_{0} \operatorname{dim}_{e} \mu \geq 2 \alpha$.

Proof. We remark that

$$
\begin{aligned}
\sum_{n \neq 0} \frac{\left(\log ^{+}|n|\right)^{s-1}}{|n|}|\widehat{\mu}(n)|^{2} & =\sum_{k=0}^{\infty} \sum_{|n| \in\left[2^{k}, 2^{k+1}\right)} \frac{\left(\log ^{+}|n|\right)^{s-1}}{|n|}|\widehat{\mu}(n)|^{2} \\
& \leq c \sum_{k=0}^{\infty} \frac{\left(\log 2^{k}\right)^{s-1}}{2^{k}}\left\|\mu * D_{2^{k+1}}\right\|_{2}^{2} \\
& \leq c \sum_{k=0}^{\infty} \frac{k^{s-1}}{2^{k}}\left\|D_{2^{k+1}}\right\|_{2,2,-\alpha}^{2}
\end{aligned}
$$

Substituting the bound for the norm of the Dirichlet kernel from the lemma and simplifying shows that

$$
\sum_{n \neq 0} \frac{\left(\log ^{+}|n|\right)^{s-1}}{|n|}|\widehat{\mu}(n)|^{2} \leq \sum_{k=0}^{\infty} \frac{k^{s-1}}{2^{k}} 2^{k} k^{-2 \alpha}
$$

and this is finite provided $s<2 \alpha$.

We will use the following elementary inequalities to obtain a partial converse to this result.

Lemma 3.5. Suppose Fine $_{0} \operatorname{dim}_{e} \mu=s>1$. Then

$$
\sum_{|n|=N}^{\infty} \frac{|\widehat{\mu}(n)|^{2}}{|n|} \leq O(\log N)^{1-s} \text { and } \sum_{|n| \leq N}|\widehat{\mu}(n)|^{2} \leq O\left(N(\log N)^{1-s}\right) \text {. }
$$


PRoof. In fact, the second inequality follows directly from the first. The first can be obtained by simply noting that

$$
\sum_{n=N}^{\infty} \frac{|\widehat{\mu}(n)|^{2}}{|n|} \leq \sum_{n=N}^{\infty} \frac{\left(\log ^{+}|n|\right)^{s-1}}{|n|}|\widehat{\mu}(n)|^{2}\left(\log ^{+}|n|\right)^{1-s} \leq O(\log N)^{1-s} .
$$

Proposition 3.6. Suppose Fine $\operatorname{dim}_{e} \mu=s>1$. If $\alpha=(s-1) / 2$, then there is a constant $c$ such that

$$
\|\mu * f\|_{2} \leq c\|f\|_{2,2,-\alpha}
$$

whenever $f$ is a Dirichlet kernel or the characteristic function of an interval.

Proof. For Dirichlet kernels the proof follows immediately from the second inequality above and the estimates we have for the Lorentz-Zygmund norms of these kernels.

So assume $f=\chi_{I}$ for $I=[a, b]$. As $|\hat{f}(n)|=\left|\left(e^{i n b}-e^{i n a}\right) / n\right|$ when $n \neq 0$, an application of the mean value theorem shows we need to bound

$$
\sum_{n \neq 0}|\widehat{\mu}(n)|^{2}\left|\left(e^{i n b}-e^{i n a}\right) / n\right|^{2} \leq \sum_{n \neq 0}|\widehat{\mu}(n)|^{2}\left|\left(e^{i n b}-e^{i n a}\right) / n\right| b-a .
$$

Break up the sum at $N=\lceil 1 /(b-a)\rceil$. For the sum for $|n| \leq N$ we apply the mean value theorem again and use the bound for $\sum_{|n| \leq N}|\widehat{\mu}(n)|^{2}$ which was given in the previous lemma. To handle the sum for $|n|>N$, we note that $\left|e^{i n b}-e^{i n a}\right| \leq 2$ and then use the lemma to bound $\sum_{|n|=N}^{\infty}|\widehat{\mu}(n)|^{2} /|n|$. Thus both parts of the sum are dominated by

$$
c|b-a|(1-\log |b-a|)^{1-s},
$$

which is comparable to $\left\|\chi_{I}\right\|_{2,2,-\alpha}^{2}$.

These results can be extended to a larger class of functions.

Lemma 3.7. Suppose there is a constant c such that for all intervals I,

$$
\left\|\mu * \chi_{I}\right\|_{2} \leq c\left\|\chi_{I}\right\|_{2,2,-\alpha} .
$$

Put $\beta=\alpha-1 / 2$. There is a constant c such that for all monotonic, integrable functions $f$,

$$
\|\mu * f\|_{2} \leq c\|f\|_{2,2,-\beta} .
$$

Proof. Suppose $f$ is a positive, decreasing, step function, say $f=$ $\sum_{k=1}^{N} a_{k} \chi_{I_{k}}$, where $a_{k}$ are decreasing and $I_{k}$ are disjoint, adjacent intervals. 
Put $b_{k}=a_{k}-a_{k+1}, b_{N}=a_{N}$ and $U_{k}=\bigcup_{j=1}^{k} I_{j}$, so $f^{*}=\sum b_{k} \chi_{U_{k}}$. One can easily check that

$$
\|f\|_{2,1,-\alpha}=\sum_{k} b_{k}\left\|\chi_{U_{k}}\right\|_{2,1,-\alpha} .
$$

Also, by assumption,

$$
\|\mu * f\|_{2} \leq \sum b_{k}\left\|\mu * \chi_{U_{k}}\right\|_{2} \leq c \sum b_{k}\left\|\chi_{U_{k}}\right\|_{2,2,-\alpha} .
$$

The inclusions of the Lorentz-Zygmund spaces imply that for any function $g$, $\|g\|_{2,2,-\alpha} \leq c\|g\|_{2,1,-\alpha}$ and $\|g\|_{2,1,-\alpha} \leq c\|g\|_{2,2,-\beta}$. Combining these facts yields

$$
\|\mu * f\|_{2} \leq c\|f\|_{2,2,-\beta} .
$$

By taking limits we can extend this result to any positive, decreasing function. The general monotonic case can be handled by considering the positive and negative parts of the function.

Putting the two preceeding results together gives,

Corollary 3.8. Suppose Fine $\operatorname{dim}_{e} \mu=s>2$. If $\beta=s / 2-1$, then there is a constant $c$ such that

$$
\|\mu * f\|_{2} \leq c\|f\|_{2,2,-\beta}
$$

for all monotonic, integrable functions $f$.

We can also obtain a sufficient condition for positive fine 0-energy dimension.

COROLlary 3.9. Let $0<\alpha<1$ and suppose there is a constant $c$ such that

$$
\left\|\mu * \chi_{I}\right\|_{2} \leq c\left\|\chi_{I}\right\|_{2,2,-\alpha}
$$

for all intervals $I$. Then $\mu$ has positive fine 0-energy dimension.

Proof. For $\varepsilon>0$ set $f=|x|^{-1 / 2}|\log | x|/ 3|^{-\varepsilon}$ and put $\beta=1 / 2-$ $\alpha$. As $f$ is decreasing near 0 and smooth away from 0 , it is easy to check $f \in L^{2,2}(\log L)^{-\beta}$ provided $\varepsilon<1-\alpha$. With the notation of Proposition 2.9, $K_{f} \sim|\log | x|/ 3|^{1-2 \varepsilon}$ since $f^{\prime}(x) \sim|x|^{-3 / 2}|\log | x|/ 3|^{-\varepsilon}$. Moreover, by Cor. 2.8,

$$
\begin{aligned}
\iint|\log | x-y|/ 3|^{1-2 \varepsilon} d \mu(x) d \mu(y) & \sim \sum_{n \neq 0} \frac{\left(\log ^{+}|n|\right)^{-2 \varepsilon}}{|n|}|\widehat{\mu}(n)|^{2}+|\widehat{\mu}(0)|^{2} \\
& \sim\|f * \mu\|_{2}^{2} .
\end{aligned}
$$


The previous proposition implies that $\|f * \mu\|_{2}<\infty$, consequently, Fine $_{0}$ $\operatorname{dim}_{e} \mu \geq 2 \alpha$.

\subsection{Orlicz-improving measures}

Definition 3.3. Similarly, a measure will be said to be Orlicz-improving if it maps an Orlicz space $L^{\Phi} \equiv\left\{f: \int \Phi(|f|)<\infty\right\}$, for some $\Phi$ satisfying $\Phi(x) / x^{2} \rightarrow 0$, into (the smaller space) $L^{2}$.

Proposition 3.10. Suppose $\alpha>0$. If $\widehat{\mu}^{2}$ belongs to the Orlicz space $L^{A}$, where

$$
A(t)= \begin{cases}\int_{0}^{t} \exp \left(-(c x)^{-1 / \alpha}\right) d x & \text { for } t \leq 1 \\ \infty & \text { for } t>1\end{cases}
$$

(for some constant $c>0$ ), then $\mu * L^{\Phi} \subseteq L^{2}$ for a Young's function $\Phi(t) \sim$ $t^{2}(\log (2+t))^{-\alpha}$.

Proof. Given $\alpha>0$, there is some $k>1$ such that $\phi(x)=x(\log (k+x))^{-\alpha}$ is increasing (to infinity). Hence $\Phi(t)=\int_{0}^{t} \phi(x) d x$ is a Young's function and it is comparable to $t^{2}(\log (2+t))^{-\alpha}$. Because $\phi(x) / x$ is decreasing, the Hausdorff-Young inequality for Orlicz spaces ([11]) implies

$$
\|\hat{f}\|_{L^{\widetilde{\Phi}}} \leq 2\|f\|_{L^{\Phi}}
$$

where

$$
\widetilde{\Phi}=\int_{0}^{t} \frac{d x}{\phi^{-1}(1 / x)} .
$$

Also, if $A^{*}$ is the conjugate Young's function to $A$, then Holder's inequality gives

$$
\|\mu * f\|_{2}^{2}=\left\|\widehat{\mu}^{2} \hat{f}^{2}\right\|_{1} \leq 2\left\|\widehat{\mu}^{2}\right\|_{L^{A}}\left\|\hat{f}^{2}\right\|_{L^{A^{*}}}=2\left\|\widehat{\mu}^{2}\right\|_{L^{A}}\|\hat{f}\|_{L^{B}}
$$

for $B(t)=A^{*}\left(t^{2}\right)$. If we put $a(x)=\exp -(c x)^{-1 / \alpha}$, then $A^{*}(t)=\int_{0}^{t} a_{+}^{-1}(x) d x$ where $a_{+}^{-1}(x)=a^{-1}(x)$ if $x \leq 1$ and 1 else. Thus, $B(0)=0$ and $B(t) \sim t^{2} \sim$ $\widetilde{\Phi}(t)$ for large $t$. It is a routine calculation to check that $B^{\prime}(t)=2 t a^{-1}(t) \leq$ $C \widetilde{\Phi}^{\prime}(t)$ for small $t$, hence $B(t) \leq C \widetilde{\Phi}(t)$ for some constant $C$. It follows that

$$
\|\mu * f\|_{2}^{2} \leq 2 C\left\|\widehat{\mu}^{2}\right\|_{L^{A}}\|\hat{f}\|_{L^{\tilde{\Phi}}} \leq 4 C\left\|\widehat{\mu}^{2}\right\|_{L^{A}}\|f\|_{L^{\Phi}}
$$

and thus if $\widehat{\mu}^{2} \in L^{A}$, then $\mu * L^{\Phi} \subseteq L^{2}$.

COROLlary 3.11. Suppose $\mu$ is a measure on $\mathrm{T}$. If there is some $c>0$ such that

$$
\sum|\widehat{\mu}(n)|^{2} \exp \left(-\left(c|\widehat{\mu}(n)|^{2}\right)^{-1 / \alpha}\right)<\infty
$$


then $\mu * L^{2,2}(\log L)^{-\alpha / 2} \subseteq L^{2}$

REMARK 3.1. A similar condition for Lorentz-improving measures is given in [7].

Proof. Without loss of generality $\|\mu\| \leq 1$. Then

$$
\begin{aligned}
\sum_{n} A\left(|\widehat{\mu}(n)|^{2}\right) & =\sum_{n} \int_{0}^{|\widehat{\mu}(n)|^{2}} \exp \left(-(c x)^{-1 / \alpha}\right) d x \\
& \leq \sum|\widehat{\mu}(n)|^{2} \exp \left(-\left(c|\widehat{\mu}(n)|^{2}\right)^{-1 / \alpha}\right)
\end{aligned}
$$

and this is finite by assumption. The conclusion follows since $L^{2,2}(\log L)^{-\alpha / 2}$ coincides with the Orlicz space $L^{\Phi}$ for $\Phi(t) \sim t^{2}(\log (2+t))^{-\alpha}$.

COROLlaRY 3.12. Suppose $\mu$ is a measure supported on a compact subset of $\mathrm{R}^{n}$. If

$$
\int_{0}^{1} \exp \left(-(c x)^{-1 / \alpha}\right) m\left\{z:|\widehat{\mu}(z)|^{2} \geq x\right\} d x<\infty,
$$

then $\mu * L^{\Phi} \subseteq L^{2}$

PRoof. In this case we can observe that

$$
\begin{aligned}
\int A\left(|\widehat{\mu}(z)|^{2}\right) d z & =\int_{-\infty}^{\infty} \int_{0}^{|\widehat{\mu}(z)|^{2}} \exp \left(-(c x)^{-1 / \alpha}\right) d x d z \\
& =\int_{0}^{1} \exp \left(-(c x)^{-1 / \alpha}\right) D(\sqrt{x}) d x
\end{aligned}
$$

where $D(x)$ is the distribution function of $\widehat{\mu}, D(x)=m\{z:|\widehat{\mu}(z)| \geq x\}$.

EXAMPLE 3.1. An example of such a measure is given in [1]. There it is shown that if $\mu=\chi \sigma$ where $\sigma$ denotes arc length measure on the curve $(t, \gamma(t))$ for $\gamma(t)=\exp -\left(t^{-p}\right)$ and $\chi \in C_{0}^{\infty}\left(\mathbf{R}^{2}\right)$, then the distribution function of $\widehat{\mu}$ satisfies $D(x) \leq C \gamma^{\prime}(x) \gamma(x / C)^{-2}$ and $\mu * L^{\Phi} \subseteq L^{2}$ for $\Phi \sim t^{2}\left(1+\log _{+} t\right)^{-2 / p}$. With this estimate on the distribution function our methods also give the conclusion that $\mu * L^{\Phi} \subseteq L^{2}$ as the hypothesis of the corollary above is satisfied with $\alpha=p / 2$.

Although these measures are 'flat', it follows that they necessarily have positive Fine 0-energy dimension. 


\subsection{Lipschitz-like properties}

In [10] it is shown that positive energy dimension is equivalent to the measure being Lipschitz. There is a partial analogue of that fact for the fine 0-energy dimension. We will say a measure $\mu$ is $\log -\operatorname{Lip}(\alpha)$ if

$$
|\mu[x, x+h]| \leq c|\log h|^{-\alpha} \quad \text { for all } \quad h>0 .
$$

Using classical results, such as can be found in [2] and [4], it can be shown that a measure $\mu$ is $\log$ - $\operatorname{Lip}(\alpha)$ for $\alpha>0$ if

$$
\sum_{|n|=N}^{\infty} \frac{|\widehat{\mu}(n)|}{|n|} \leq c(\log N)^{-\alpha},
$$

and that if $\mu$ is $\log -\operatorname{Lip}(\alpha)$ for $\alpha>1$, then

$$
\sum_{|n|=N}^{\infty} \frac{|\widehat{\mu}(n)|^{2}}{|n|} \leq c(\log N)^{1-\alpha} .
$$

With these facts one can easily prove that if $\mu$ is $\log -\operatorname{Lip}(\alpha)$ for some $\alpha>1$, then Fine $\operatorname{dim}_{e} \mu \geq \alpha-1$, while if Fine $\operatorname{dim}_{e} \mu=s>2$, then $\mu$ is Log$\operatorname{Lip}(\alpha)$ for $\alpha \geq(s-1) / 2$.

\section{REFERENCES}

1. Bak, J-G., McMichael, J. and Oberlin, D., Convolution estimates for some measures on flat curves, J. Funct. Anal. 101 (1991), 81-96.

2. Bary, N., A Treatise on Trigonometric Series, Vol. 1, MacMillan Co., New York (1964).

3. Bennett, C., and Rudnick, K., On Lorentz-Zygmund spaces, Dissertationes Math. 175 (1980), $1-72$.

4. Boas, R., Fourier series with positive coefficients, J. Math. Anal. Appl. 17 (1967), 463-483.

5. Falconer, K., Fractal Geometry; Mathematical Foundations and Applications, Wiley and Sons, Chichester (1990).

6. Falconer, K., Techniques in Fractal Geometry, Wiley and Sons, Chichester (1997).

7. Grinnell, R., and Hare, K., Lorentz-improving measures, Illinois J. Math. 38 (1994), 366-389.

8. Hare, K., and Roginskaya, M., A Fourier series formula for energy of measures with applications to Riesz products, Proc. Amer. Math. Soc. 131 (2003), 165-174.

9. Hare, K., and Roginskaya, M., Energy of measures on compact Riemannian manifolds, Studia Math. 159 (2003), 291-314.

10. Hare, K., and Roginskaya, M., $L^{p}$-improving properties of measures of positive energy dimension, Colloq. Math. 102 (2005), 73-86.

11. Jodeit, M., and Torchinsky, A., Inequalities for Fourier transforms, Studia Math. 37 (1971), 245-276.

12. Kahane, J-P., Ensembles Parfaits et Series Trigonometriques, Hermann, Paris (1963).

13. Mattila, P., Geometry of Sets and Measures in Euclidean Spaces, Cambridge Univ. Press, Cambridge (1995). 
14. Rogers, C., Hausdorff Measures, Cambridge Univ. Press, London (1970).

15. Sagher, Y., An application of interpolation theory to Fourier series, Studia Math. 61 (1972), $168-181$.

16. Sjölin, P., and Soria, F., Some remarks on restrictions of the Fourier transform for general measures, Publ. Mat. 43 (1999), 655-664.

DEPT. OF PURE MATHEMATICS

UNIVERSITY OF WATERLOO

WATERLOO, ONT.

CANADA N2L 3G1

E-mail: kehare@uwaterloo.ca

DEPT. OF MATHEMATICS

CHALMERS TH AND GOTEBORG UNIVERSTIY

EKLANDAGATAN 86

SE 41296 GÖTEBORG

SWEDEN

E-mail: maria@math.chalmers.se
DEPT. OF MATHEMATICS

UNIVERSITY OF DELHI

DELHI 110007

INDIA

E-mail: pmohanty@maths.du.ac.in 